\title{
Modelo del Pedagogical content Knowledge aplicado en probabilidad para la educación media
}

\section{Pedagogical content Knowledge model applied in probability for secondary}

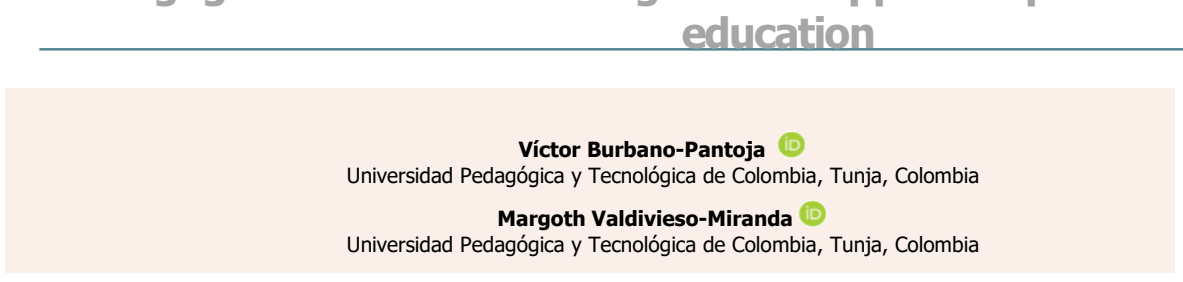

\section{Resumen}

Objetivo: Explorar el Pedagogical Contente Knowledge (PCK) de profesores que enseñan el tema de probabilidad en la educación media en Tunja. Método: Se siguió un enfoque cualitativo de investigación centrado en un estudio de casos múltiple y un análisis de contenido sobre la práctica pedagógica de una muestra seleccionada por criterios como: potencial conocimiento del tema, experiencia docente y posibilidad de observar la práctica de aula, entre otros. La información fue recolectada mediante una entrevista semi-estructurada, diario de campo, situaciones problema de probabilidad y materiales docentes; los datos textuales fueron codificados de forma selectiva y axial e interpretados a la luz del círculo hermenéutico y triangulación de fuentes de información. Resultados: Éstos evidencian la emergencia de tres categorías de conocimiento requeridas por el docente para enseñar probabilidad: conocimiento del contenido por enseñar, conocimiento de estrategias instruccionales y conocimiento de las formas como el alumno aprende este tema. Discusión y Conclusiones: Los hallazgos evidencian que es perentorio acrecentar el PCK de los docentes quienes ofrecen este tema necesario para enfrentar los desafíos inciertos del siglo XXI; se concluye que, el docente presenta un conocimiento básico del tema, bajo desarrollo de su PCK y poca comprensión del concepto de probabilidad.

Palabras clave: Educación media, investigación cualitativa, Pedagogical Content Knowledge, Probabilidad.

\section{Abstract}

Objective: To explore the Pedagogical Contente Knowledge (PCK) of teachers who teach the subject of probability in middle education of Tunja. Method: A qualitative research approach centered on a multiple case study and a content analysis on the pedagogical practice of a sample selected by criteria such as: potential knowledge of the subject, teaching experience and the possibility of observing classroom practice, among others, was followed. The information was collected through a semistructured interview, a field diary, probability problem situations and teaching materials; the textual data were coded selectively and axially and interpreted in the light of the hermeneutical circle and triangulation of information sources. Results: These evidence the emergence of three categories of knowledge required by the teacher to teach probability: knowledge of the content to be taught, knowledge of instructional strategies, and knowledge of the ways in which the student learns this topic. Discussion y Conclusion: The findings show that it is imperative to increase the PCK of teachers who offer this necessary subject to face the uncertain challenges of the 21st century; it is concluded that, the teacher presents a basic knowledge of the subject, under development of his PCK and little understanding of the concept of probability.

Keywords: Midle education, pedagogical content knowledge, probability, qualitative research
Open Access:

ISSN: $0124-2121$

E-ISSN: $2665-2420$

ARTÍ́CULO DE INVESTIGACIÓN CIENTÍFICA
Copyright $\odot$ ) By Educación y Humanismo

Editor:

Dhayana Fernández Matos

Correspondencia: victor.burbano@uptc.edu.co

Recibido: 13-01-2021 Aceptado: $28-07-2021$ En línea desde: 08-10-2021

Cómo citar este artículo (APA): Burbano-Pantoja, V. y Valdivieso-Miranda, M. (2021). Modelo del Pedagogical content Knowledge aplicado en probabilidad para la educación media. Educación y Humanismo, 23(41), 234-253. https://doi.org/10.17081/eduhum.23.41.4321 


\section{Introducción}

La educación puede entenderse como un proceso de transformación cultural y de humanización de los individuos (Torres, Cifuentes \& Espejo, 2020); en este sentido, la formación del profesor es un tema fundamental dentro del sistema educativo en diversos países; los aspectos referidos a la calidad de la educación dependen, en un alto porcentaje, del nivel de formación alcanzado por el docente y de los conocimientos que explicita durante el ejercicio de su práctica pedagógica. Un mayor grado de preparación del profesor puede contribuir, en gran medida, al mejoramiento de los procesos educativos; en particular, los referidos a enseñar y aprender las matemáticas desde un punto de vista más humanista. Para este propósito, se deben aprovechar los resultados logrados por la investigación educativa y el nuevo conocimiento que se pueda generar en forma de respuestas a problemas o cuestionamientos sobre el contenido curricular cuando éste se establece en el sistema escolar o el conocimiento que resulte de considerar el proceso enseñanzaaprendizaje como objeto de estudio (Burbano, 2017) de manera general o al abordar tópicos específicos dentro de las distintas asignaturas que conforman el currículo escolar, como es el caso de las matemáticas, la estadística o la probabilidad.

En la actualidad, la determinación de los conocimientos requeridos por el profesor para la enseñanza de tópicos de probabilidad es una de las problemáticas que mayor interés ha suscitado en la comunidad de investigadores tanto a nivel internacional como nacional. Paradigmas de investigación referentes al conocimiento pedagógico, conocimiento didáctico del contenido (Shulman, 1987) y conocimiento matemático para la enseñanza (Ball, Thames \& Phelps, 2008), señalan importantes avances investigativos en esta dirección. En este trabajo investigativo se exploró acerca del PCK de profesores quienes imparten el tema de probabilidad en el nivel de educación media. El objeto de estudio fue abordado desde la perspectiva de investigación focalizada en el denominado 'conocimiento profesional' del profesor de matemáticas, particularmente de probabilidad y estadística.

Por otra parte, en los últimos 35 años, de forma progresiva, el tema de probabilidad se ha incluido en los currículos de matemáticas del nivel pre universitario de numerosos países, por ser un tema con potenciales aplicaciones en ámbitos como: la educación escolar y universitaria, la academia, la investigación científica, la industria, la política y la vida cotidiana, entre otros (Swenson, 1998; Batanero, 2020); en consecuencia, es conveniente incluirla en el currículo y enseñarla desde el nivel de la educación primaria y secundaria hasta el universitario (NCTM, 2000; MEN, 2003; Vásquez, 2014); además, el PCK en el tema ha sido poco investigado tanto en el ámbito internacional como nacional. En este contexto, la pregunta que orientó el proceso investigativo fue ¿Cuáles conocimientos manifiesta el docente para enseñar el tema de probabilidad en su práctica pedagógica?

En seguida se presentan aspectos referidos al PCK, al tema de probabilidad, la

práctica pedagógica y los antecedentes tanto a nivel nacional como internacional. Hace 
aproximadamente tres décadas, el PCK fue definido por Shulman (1987) como: una mezcla, amalgama o combinación entre la materia por enseñar y su didáctica por medio de la cual se llega a una "comprensión de cómo temas específicos y problemas se adaptan, se organizan y se representan a las distintas capacidades e intereses de los estudiantes, y se exponen para su enseñanza" (p. 8). En este contexto, el PCK es un constructo que se estructura en la mente del profesor y se utiliza para enseñar un determinado tema. Adicionalmente, el PCK incluye "formas útiles de representación del contenido, así como las más poderosas y potentes ilustraciones, analogías, explicaciones, ejemplos y demostraciones de forma reducida, las maneras de representar y formular la materia de modo que la hagan comprensible a otros" (Shulman, 1987, p. 9); es decir, el PCK recoge diversas estrategias didácticas para favorecer el aprendizaje estudiantil, las cuales Shulman denomina estrategias instruccionales.

El PCK se constituye en la principal de las siete categorías del conocimiento base para la enseñanza (Burbano, Valdivieso \& Aldana, 2017) y a su vez, el PCK contempla tres categorías que lo distinguen: i) el conocimiento del contenido a enseñar, ii) el conocimiento de estrategias y representaciones instruccionales, y iii) el conocimiento sobre el aprendizaje del estudiante. La primera hace referencia al conocimiento de la materia específica o tópico a enseñar relacionado con el currículo propuesto por la institución educativa y con las políticas del sistema escolar; este se define como la "cantidad y organización de conocimiento per se en la mente del profesor" (Shulman, 1986, p. 9). Las representaciones instruccionales (RI) corresponden a "formas de expresar, exponer, escenificar o de representar de otra manera ideas, de suerte que los que no saben puedan llegar a saber, los que no entienden puedan comprender y discernir, y los inexpertos puedan convertirse en expertos" (Shulman, 1986, p. 9); éstas son representaciones usadas para enseñar un tópico específico y pueden concebirse como elementos utilizados por el profesor para favorecer en el alumno la generación del conocimiento, la construcción y el establecimiento de relaciones. Entre ellas están: dibujos, representaciones gráficas y simbólicas provenientes de los libros o de la mente del profesor, modelos que posibiliten comunicar a los alumnos algo enseñable, actividades, preguntas, analogías, exposiciones verbales, diagramas, simulations, metáforas, dramatizaciones y análisis de contenido, entre otras.

El conocimiento sobre el aprendizaje del estudiante incluye las estrategias con las que el profesor ayuda a los alumnos en su aprendizaje, recoge el conocimiento de los errores conceptuales, creencias y concepciones frecuentes en el estudiante, y las condiciones de instrucción que sean necesarias para lograr una transformación de dichas concepciones de forma pertinente (Shulman, 1986). Se enfatiza que este conocimiento ha de favorecer la comprensión de los conceptos en el alumno y que el profesor ha de conocer la génesis y evolución del proceso cognitivo del estudiante en relación a su edad, grado, experiencia y escolaridad, sus motivaciones intrínsecas y extrínsecas, expectativas y dificultades enmarcadas en una perspectiva del aprendizaje de las matemáticas o la probabilidad y del tópico específico a desarrollar en la clase (Pinto, 2010; Burbano, 2017). 
Por otro lado, la probabilidad se puede definir como la medida de la posibilidad de que un evento ocurra (Burbano \& Valdivieso, 2015). Del amplio tema de probabilidad, se decidió abordar los tópicos de estimación de probabilidades y el concepto de probabilidad. En el trabajo desarrollado, la estimación de probabilidades hizo referencia al cálculo de probabilidad de un determinado evento o a la búsqueda de una aproximación al valor de probabilidad de un suceso en concordancia con alguna de las concepciones de probabilidad. El conocimiento del contenido probabilístico corresponde a los tópicos del conocimiento probabilístico necesarios para la enseñanza y para resolver problemas relacionados con la probabilidad. En el nivel pre universitario, los contenidos se desprenden de los estándares curriculares sugeridos por el Ministerio de Educación Nacional -MEN- en el año 2003; este conocimiento puede incluir: el concepto de probabilidad desde diferentes concepciones, espacio muestral, eventos y su probabilidad, heurísticas y sesgos, probabilidad condicional y variables aleatorias con su distribución, entre otros (MEN, 2003).

En cuanto al concepto de probabilidad, un análisis de contenido sobre diversas fuentes documentales (Bardín, 1991) permitió la elaboración de un texto sobre el desarrollo histórico-epistemológico de tal concepto. Este desarrollo incluyó cuatro momentos: la prehistoria de la probabilidad que recoge acontecimientos desde la antigüedad hasta Pascal, los siglos XVII y XVIII en los cuales se da la emergencia del concepto de probabilidad, las últimas décadas del siglo XVIII y las primeras del siglo XIX en las que se genera la teoría clásica, y las primeras tres décadas del siglo XX periodo en el cual se hace la formulación axiomática (Burbano \& Valdivieso, 2015); allí, se recogen las principales concepciones en torno al concepto (Hacking, 1995), entre ellas: la concepción clásica (Laplace, 1812/1985), frecuencial (Mises, 1928) y axiomática (Kolmogórov, 1933); las cuales han de ser apropiadas por el profesor a fin de consolidar o ampliar su conocimiento probabilístico necesario para orientar de manera pertinente el proceso enseñanza-aprendizaje del tema de probabilidad (Burbano \& Valdivieso, 2020).

En referencia a la práctica pedagógica, desde un punto de vista operativo, ésta puede entenderse como 'la actividad del profesor' constituida por tres fases: pre-activa, interactiva y post-activa (Jackson, 1975) susceptible de analizarse de manera cualitativa (Le Compte \& Goetz, 1982); en este contexto, más allá de los conocimientos disciplinares que el profesor posee, lo fundamental es la manera cómo el docente realiza su acción pedagógica en el aula (Ponte, Quaresma \& Branco, 2012; Burbano, 2017); así entonces, la práctica pedagógica ha de conceptualizarse como sinónimo de acción e interacción en el aula, lo que el profesor hace con el saber para que sus estudiantes lo aprendan (Sánchez, 2011). Desde un enfoque cognitivo, la práctica del ejercicio profesoral se centra en la actividad docente (Llinares, 2000) en el marco de su conocimiento como profesional de la educación; desde una perspectiva sociocultural, la mencionada práctica, se relaciona con una imagen o trayectoria de aquello que ocurre en los instantes de interacción, encuentro y compartir entre el docente, el estudiante y el saber por asimilar; en estas circunstancias, el docente utiliza distintos instrumentos o estrategias con determinados significados, los cuales posibilitan una caracterización de tal práctica (Lave \& Wenger, 1991). Desde una mirada holística, en este 
trabajo, se buscaba explorar la forma como el profesor integraba el concepto de probabilidad focalizado en las concepciones clásica, frecuencial y axiomática, algunas representaciones instruccionales específicas y esquemas alusivos, los cuales posibilitarían un análisis de su práctica pedagógica y la exploración referida la comprensión de este concepto por parte del profesor y su forma de impartirlo a sus estudiantes.

En cuanto a los a antecedentes, desde su gestación, el PCK fue evolucionando de forma progresiva sin perder vigencia, transformándose y constituyéndose en un marco teórico con buen potencial para el desarrollo de la investigación educativa en diversos campos del conocimiento tales como ciencias, matemáticas y estadística, entre otros (Burbano, 2017). Además, este marco ha servido de inspiración para la generación de diversos modelos de conocimiento del profesor destinados a la enseñanza de distintas materias en el currículo escolar (Burbano, et al., 2017); sin embargo, el PCK en probabilidad ha sido poco investigado tanto a nivel internacional como nacional. Su fuerte relación con las didácticas específicas, ha llevado a los investigadores a considerarlo como objeto de estudio en diferentes áreas de conocimiento y un recurso importante para generar programas de cualificación docente en distintos países (Appova \& Taylor, 2020).

El PCK ha sido investigado en una amplia gama de temas en los campos de ciencias, matemáticas, estudios sociales, inglés, educación física, medicina, comunicación, tecnología, ingeniería, estadística, enfermería, derecho, negocios, música, entre otros; en estadística se destacan los trabajos de Batanero, Sorto, Watson, Burguess, Noll, Watson, Callingham y Done, Pinto y Estrella (Pinto, 2010; Burbano, 2017; Appova \& Taylor, 2020), por mencionar algunos. No obstante, el número de trabajos relacionados con el PCK en educación estadística aún puede considerarse bajo y mucho menor en la enseñanza de la probabilidad (Venkat \& Adler, 2020). En el ámbito internacional, entre los pocos trabajos realizados sobre este constructo en probabilidad se encuentran el de Swenson, Watson, Callingham y Watson. El primero estudia el conocimiento de la materia y el PCK sobre el tópico de probabilidad mediante un estudio de casos con cuatro profesores de secundaria en Estados Unidos con el fin de identificar algunas relaciones focalizadas en la forma cómo los docentes enseñaban el mencionado tópico (Swenson, 1998). El segundo analiza el conocimiento de profesores australianos para la enseñanza de la probabilidad y la estadística usando el marco teórico de Shulman (Watson, 2001). El tercero desarrolla un proyecto de formación profesional que valora el PCK de varios profesores en cuatro niveles jerárquicos en concordancia con el modelo de conocimiento de Shulman (Callingham \& Watson, 2011).

Por otra parte, el PCK sirvió para generar el modelo denominado MKT o de conocimiento matemático para la enseñanza (Ball, et al., 2008), el modelo de Schoenfeld \& Kilpatrick (2008) centrado en las relaciones interpersonales entre docente y estudiantes al concebir el proceso enseñanza-aprendizaje como una actividad socializadora influenciada por la interacción continua entre los actores intervinientes en tal proceso, el modelo de Godino (2009) que recoge la complejidad del mencionado proceso al amalgamar los modelos de Shulman, Ball y Schoenfeld, y proponer el Enfoque Onto-Semiótico (EOS). En este 
contexto, se pueden mencionar el trabajo de Vásquez (2014) referido a la evaluación del conocimiento didáctico-matemático para enseñar probabilidad de los profesores de educación primaria en activo y el estudio efectuado por Gómez (2014) focalizado en la evaluar y desarrollar el conocimiento matemático para enseñar el tema de probabilidad en futuros profesores de educación primaria. Finalmente, en el campo de la probabilidad aún se están generando aproximaciones orientadas a la construcción de un modelo propio con las propuestas atribuidas a Jones, Langrall, Thornton \& Mogill (1997) y Even \& Kvatinsky (2010); en estas circunstancias, de forma alternativa ha sido conveniente adoptar modelos para enseñar matemáticas como el EOS o el de Conocimiento Didáctico matemático (CDM) expuesto por diversos autores (Pincheira, Vásquez \& Giacomone, 2021).

Trabajo investigativos realizados en los últimos cinco años y focalizados en la didáctica de la probabilidad en el ámbito colombiano son escasos (Rodríguez, 2021), estudios abordados desde el modelo del PCK en probabilidad son muy pocos (Burbano, 2017) e investigaciones que involucren el PCK y su metodología se centre en estudios no se han documentado (Burbano \& Valdivieso, 2020; Rodríguez, 2021). Antecedentes como los expuestos, ilustraron la necesidad de explorar el PCK en el contexto colombiano con profesores de matemáticas del nivel pre universitario centrado en enseñar el tema; asimismo, se generan oportunidades de mejora en los procesos de formación docente o de actualización del profesorado en el ámbito de la probabilidad. En futuras investigaciones se pueden abordar aspectos asociados con el aprendizaje de la probabilidad y los estilos de aprendizaje y las dificultades estudiantiles en tanto que el concepto de probabilidad se caracteriza por ser complejo (Batanero, 2020), sin presencia de la propiedad de reversibilidad en tanto que se relaciona con eventos futuros (Sriraman \& Chernoff, 2020).

\section{Método}

Un enfoque de investigación cualitativa centrado en un estudio de casos múltiple guió el estudio realizado (Rodríguez, Gil \& García, 1999). Los casos permitieron analizar de manera concisa las propias palabras de los docentes tanto de forma oral como escrita, identificar conceptos y comprensiones con base en información textual ofrecida por cuatro profesores en ejercicio, observar el contexto y la acción educativa desde una mirada integral, teniendo en cuenta su pasado y las eventualidades en las que se hallaban, comprender su forma de actuar, razonar e intervenir durante el proceso de enseñanza-aprendizaje de un tema específico (Taylor \& Bogdan, 1987; Simons, 2011).

La muestra fue seleccionada a criterio y generada a conveniencia (Hernández \& Mendoza, 2018); se conformó por cuatro profesores en activo, quienes se desempeñaban en diversas instituciones educativas en la ciudad de Tunja en Colombia y ofrecían sus clases de matemáticas en el nivel de la educación media. Los participantes fueron escogidos al considerar criterios como: i) la posibilidad de aprender de cada profesor y de lograr una mejor comprensión sobre su PCK en el tema de probabilidad (Stake, 1998), por lo tanto, el aspecto referido a si los docentes casos típicos o atípicos fue secundario, ii) su potencial 
conocimiento disciplinar sobre el tema de probabilidad a ser enseñado en el grado once de la educación formal en Colombia, iii) la experiencia docente, iv) que el profesor estuviera enseñando el tema y dispuesto tanto a colaborar como a departir sus conocimientos, iv) que el docente permitiera la observación de su práctica pedagógica e interacción con sus estudiantes en el aula, y v) que el maestro accediera a colaborar proporcionando información adicional a través de diversos instrumentos; así entonces, los casos se seleccionaron teniendo en cuenta algunas de sus virtudes y potencialidades (Yin, 2014) para enseñar el tema de probabilidad. En esta investigación no se recurre a una muestra probabilística que sea representativa de la población, como lo exigen las investigaciones de tipo cuantitativo positivista (Unangst, et al., 2020; Babbie, 2020).

Los principales instrumentos destinados a la recopilación de la información fueron una entrevista semi-estructurada (Rodríguez, et al., 1999; Pinto, 2010), observación de la práctica mediante un diario de campo (Colestock \& Sherin, 2009), dos situaciones problema centradas en las concepciones sobre probabilidad y algunos materiales del profesor tales como el programa de la asignatura, planes de clase y la libreta de apuntes del estudiante, fuentes que posibilitaron aplicar la técnica de triangulación de información (Burbano, 2017). Con la información recolectada se realizó un análisis de datos textuales por medio de exégesis textual en una forma de círculo hermenéutico (Gadamer, 1976) y métodos propios de la investigación cualitativa (Taylor \& Bogdan, 1987) tales como la codificación axial y selectiva (Strauss \& Corbin, 1998) así como la triangulación de las fuentes de información asociadas con los instrumentos (Gavira \& Osuna, 2015); además, se utilizó el software Nvivo 12 en su versión de prueba disponible en internet para luego elaborar la matriz de categorías explícita en las tabla 2 y 3 . En cuanto al manejo de la información, los participantes firmaron un consentimiento informado, en el cual se les hizo conocer el propósito del trabajo investigativo, los aspectos sobre los cuales colaborarían y se les aclaró que sus datos serían utilizados solamente para fines académicos e investigativos en correspondencia con las consideraciones éticas de investigación científica consignadas en la Ley de Habeas Data colombiana y la ley 23 de 1982 referida a derechos de autor (Torres, et al., 2020); en consecuencia, se utilizaron los siguientes seudónimos referidos a quienes participaron en el trabajo investigativo: caso 1 , caso 2 , caso 3 y caso 4.

\section{Resultados}

En este apartado se presentan los resultados asociados con este trabajo de investigación, se inicia con descripción de los participantes y se prosigue con los hallazgos referidos a las fases pre-activa, interactiva y pos-activa de la práctica pedagógica de los docentes que enseñaron el tema de probabilidad en el grado once de la educación media colombiana. 


\section{Descripción de los participantes}

El caso 1 correspondió a un profesor titulado como licenciado en matemáticas hace ocho años y desde entonces ha trabajado en la educación pre universitaria y se encuentra cursando estudios de postgrado en educación matemática. Se desempeña como profesor de contrato en la Institución Educativa del Este, IEE (seudónimo); en dicha institución privada, desarrolla su actividad pedagógica, de investigación y de orientación académica para alumnos de básica secundaria y educación media; desde hace aproximadamente cuatro años, enseña el tema de probabilidad como parte del currículo de matemáticas en los grados décimo y undécimo. El caso 2 hace referencia a otro docente titulado como matemático hace cuatro años, quien trabaja oficialmente desde hace tres años en una Institución Educativa pública IDP (seudónimo), actualmente cursa una maestría en didáctica de la matemática, ofrece el tema de probabilidad y estadística como una asignatura independiente al currículo de matemáticas en los mismos grados que el caso 1.

El caso 3 es un maestro licenciado en matemáticas y física, se graduó hace 23 años, mismo tiempo que ejerce como docente de matemáticas, tiene en su haber una maestría en educación; trabaja como profesor oficial de planta en el magisterio colombiano en la Institución Educativa del Centro IEC (seudónimo); en la IEC, desarrolla su práctica pedagógica desde el grado octavo hasta el once, en áreas como física, tecnología matemáticas y estadística (que incluye el tema de probabilidad en grado once); Además, con su apoyo académico, la IEC ha obtenido premios de reconocimiento nacional. El caso 4 hace referencia a una maestra licenciada en física y matemáticas, quien obtuvo su título hace 17 años y ese mismo lapso de tiempo ha laborado en la institución privada IER (seudónimo) ofreciendo matemáticas a los grados noveno, décimo y once, allí se trabaja la probabilidad como un tema dentro del currículo de matemáticas en los dos últimos grados; no ha cursado una maestría pero se actualiza en el tema mediante auto formación.

Así entonces, de la tabla 1 se establece que, el promedio de los años de experiencia docente es de 13 años; sin embargo, los dos primeros casos presentan en promedio seis años de experiencia, en cambio, los dos últimos han alcanzado un promedio de 20 años de experiencia; el $75 \%$ de los participantes son de género masculino y el restante $25 \%$ de género femenino; el $50 \%$ trabajan en el sector educativo oficial y el otro $50 \%$ en el privado. Solamente, el $25 \%$ tiene título de matemático y el restante $75 \%$ son licenciados en matemáticas o en física y matemáticas.

Tabla 1

Características relacionadas con los participantes en el estudio

\begin{tabular}{ccccc}
\hline Características & Caso 1 & Caso 2 & \multicolumn{1}{c}{ Caso 3 } & \multicolumn{1}{c}{ Caso 4} \\
\hline Título profesional & Licenciado en & Matemático & Licenciado en & Licenciado en \\
& Matemáticas & & matemáticas y física y \\
& & & física & matemáticas
\end{tabular}

$\begin{array}{llll}\text { Estudios de maestría } & \text { En curso no curso }\end{array}$ 


\begin{tabular}{lcccc} 
Género & Masculino & Masculino & Masculino & Femenino \\
Experiencia docente en años & 8 & 4 & 23 & $17 \mathrm{i}$ \\
$\begin{array}{l}\text { Carácter de la Institución educativa } \\
\text { donde trabaja }\end{array}$ & Privada & Pública & Privada & Pública \\
\hline
\end{tabular}

Fuente: Elaborado por los autores (2020)

\section{Fases de la práctica pedagógica en probabilidad}

El análisis textual realizado sobre la fase pre-activa o de planeación de la enseñanza reflejó la presencia de tres fuentes de información que los docentes utilizan para orientar el tema de probabilidad: el programa curricular de la asignatura, un plan de clase sobre el tema de probabilidad y la libreta de apuntes del estudiante. Los cuatro casos se basan en estos tres elementos para planear su clase de probabilidad; la triangulación de estas fuentes posibilitó la determinación de las categorías expuestas en la tabla 2.

Con base en la tabla 2, la entrevista realizada a los participantes y la exégesis textual (círculo hermenéutico) sobre el sentido que tiene para los docentes la planeación de la clase de probabilidad, se interpreta que, la planificación efectuada se orienta por los estándares curriculares generados por el Ministerio de Educación Nacional de Colombia (MEN), en los cuales se incluye el pensamiento aleatorio y los sistemas de datos basado en el trabajo con tópicos de probabilidad y estadística respectivamente para el grado once, cada docente le imprime un determinado enfoque pedagógico: constructivista, aprendizaje significativo o resolución de problemas, sea que el tema de probabilidad se aborde dentro del programa curricular de matemáticas o por separado; el plan de clase y la libreta del estudiante guardan concordancia y reflejan una prevalencia del conocimiento probabilístico centrado en la concepción intuitiva y clásica dinamizado con estrategias instruccionales focalizadas en la interacción con los juegos de azar con dados y monedas para estimar probabilidades en espacios muestrales finitos; además, se observan diversos tipos de tareas probabilísticas en tales libretas que guardan cierta relación con los aspectos reportados por Martin et al. (2021).

\section{Tabla 2}

Categorías asociadas con la fase pre-activa

\begin{tabular}{lcccc}
\hline \multicolumn{1}{c}{ Categorías } & Caso 1 & Caso 2 & Caso 3 & Caso 4 \\
\hline $\begin{array}{l}\text { C1pre. El programa Curricular } \\
\text { utilizado por el profesor incluye el }\end{array}$ & $\mathrm{Si}$ & Separado & Separado & Si \\
tema de probabilidad & & & & \\
& & & & \\
$\begin{array}{l}\text { C11pre. El programa curricular tiene Constructivista } \\
\text { tendencia }\end{array}$ & $\begin{array}{l}\text { Resolución de } \\
\text { problemas }\end{array}$ & $\begin{array}{l}\text { Aprendizaje } \\
\text { significativo }\end{array}$ & $\begin{array}{l}\text { Resolución de } \\
\text { problemas }\end{array}$
\end{tabular}


C2pre. El plan de clase es afín con los temas visibles en el plan curricular de matemáticas

C21pre. El plan de clase es afín con los temas visibles en una asignatura independiente a matemáticas

C3pre. La libreta del estudiante refleja contenidos de probabilidad intuitiva y clásica

C31pre. La libreta del estudiante evidencia el uso juegos de azar con dados, monedas y otros para estimar probabilidades

$\begin{array}{llll}\text { Si } & \text { No } & \text { No } & \text { Si } \\ \text { No } & \text { Si } & \text { Si } & \text { No } \\ \text { Si } & \text { Si } & \text { Si } & \text { Si } \\ \text { Si } & \text { Si } & \text { Si } & \text { Si }\end{array}$

Fuente: Elaborado por los autores (2020)

El análisis textual referido a la fase interactiva también denominada fase de gestión develó la emergencia de siete categorías: i) conocimiento del contenido por enseñar, ii) estrategias instruccionales utilizadas, iii) conocimiento sobre el aprendizaje estudiantil, iv) fuentes del conocimiento probabilístico, v) concepciones sobre probabilidad y su enseñanza, vi) conocimiento del currículo, y vii) comprensión del concepto de probabilidad. Las tres primeras categorías corresponden al PCK de los profesores en su práctica pedagógica.

Con base en la tabla 3, la entrevista realizada a los participantes y la exégesis textual efectuada sobre los hallazgos de su fase interactiva, permiten interpretar que, los docentes reflejan un conocimiento moderado del tema por enseñar a pesar de sus esfuerzos por auto capacitarse o de acceder a estudios de educación posgraduada; sus estrategias instruccionales dependen en alto porcentaje de sus concepciones sobre la probabilidad y las matemáticas; su conocimiento sobre el aprendizaje estudiantil guarda relación con su experiencia; no obstante, en la mayoría de ellos existen dificultades para corregir algunas heurísticas y sesgos como el de equiprobabilidad porque algunos sesgos están presentes en ellos también; por consiguiente, los profesores reflejan un PCK básico en su práctica pedagógica como consecuencia de conjugar su moderado 'conocimiento' del temas, una baja capacidad para corregir a sus estudiantes, y un creciente conocimiento de las de las estrategias instruccionales, las cuales son proporcionales a los años de experiencia docente.

\section{Tabla 3}

\section{Categorías asociadas con la fase interactiva}

\begin{tabular}{|c|c|c|c|c|}
\hline Categorías & Caso 1 & Caso 2 & Caso 3 & Caso 4 \\
\hline $\begin{array}{l}\text { C1_interactiva: } \\
\text { Conocimiento del } \\
\text { contenido por } \\
\text { enseñar }\end{array}$ & $\begin{array}{l}\text { Moderado } \\
\text { concepciones: } \\
\text { intuitiva-clásica }\end{array}$ & $\begin{array}{l}\text { Alto concepciones: } \\
\text { intuitiva-clásica } \\
\text { axiomática }\end{array}$ & $\begin{array}{l}\text { Moderado: } \\
\text { concepciones: } \\
\text { intuitiva-clásica }\end{array}$ & $\begin{array}{l}\text { Moderado: } \\
\text { concepciones: } \\
\text { intuitiva-clásica }\end{array}$ \\
\hline $\begin{array}{l}\text { C2_interactiva: } \\
\text { Estrategias } \\
\text { instruccionales }\end{array}$ & $\begin{array}{l}\text { Diagrama de Venn, } \\
\text { juegos de azar, } \\
\text { monedas }\end{array}$ & $\begin{array}{l}\text { Problemas, ejercicio- } \\
\text { formal diagramas de } \\
\text { árbol, juegos de azar, } \\
\text { dados }\end{array}$ & $\begin{array}{l}\text { Preguntas, tablas de } \\
\text { frecuencias, dados, } \\
\text { monedas }\end{array}$ & $\begin{array}{l}\text { Ejemplos, } \\
\text { problemas, } \\
\text { Ejercicios }\end{array}$ \\
\hline $\begin{array}{l}\text { C3_interactiva: } \\
\text { Conocimiento del } \\
\text { aprendizaje } \\
\text { estudiantil }\end{array}$ & $\begin{array}{l}\text { Corrige algunas } \\
\text { heurísticas pero } \\
\text { tiene otras. } \\
\text { Motiva al estud. }\end{array}$ & $\begin{array}{l}\text { Corrige algunos } \\
\text { errores de cálculo e } \\
\text { interpretación }\end{array}$ & $\begin{array}{l}\text { Corrige algunas } \\
\text { heurísticas pero tiene } \\
\text { otras }\end{array}$ & $\begin{array}{l}\text { Motiva al } \\
\text { estudiante } \\
\text { y le corrige } \\
\text { algunos sesgos }\end{array}$ \\
\hline
\end{tabular}




\begin{tabular}{|c|c|c|c|c|}
\hline $\begin{array}{l}\text { C4_interactiva: } \\
\text { Fuentes del } \\
\text { conocimiento } \\
\text { probabilístico }\end{array}$ & $\begin{array}{l}\text { Recibió dos cursos } \\
\text { para estadística y } \\
\text { probabilidad }\end{array}$ & $\begin{array}{l}\text { Recibió tres cursos } \\
\text { para estadística y } \\
\text { probabilidad }\end{array}$ & $\begin{array}{l}\text { Recibió un curso } \\
\text { integrado de } \\
\text { estadística y } \\
\text { probabilidad }\end{array}$ & $\begin{array}{l}\text { Recibió un curso } \\
\text { integrado de } \\
\text { estadística y } \\
\text { probabilidad }\end{array}$ \\
\hline $\begin{array}{l}\text { C5_interactiva: } \\
\text { Concepciones sobre } \\
\text { probabilidad }\end{array}$ & $\begin{array}{l}\text { Probabilidad clásica, } \\
\text { equiprobabilidad } \\
\text { espacios finitos }\end{array}$ & $\begin{array}{l}\text { Probabilidad clásica, } \\
\text { espacios finitos e } \\
\text { infinitos }\end{array}$ & $\begin{array}{l}\text { Probabilidad clásica, } \\
\text { equiprobabilidad } \\
\text { espacios finitos }\end{array}$ & $\begin{array}{l}\text { Probabilidad } \\
\text { intuitiva-clásica, } \\
\text { espacios finitos }\end{array}$ \\
\hline $\begin{array}{l}\text { C6_interactiva: } \\
\text { Conocimiento del } \\
\text { currículo }\end{array}$ & $\begin{array}{l}\text { Adecuado a su } \\
\text { formación } \\
\text { profesional }\end{array}$ & $\begin{array}{l}\text { Adecuado a su } \\
\text { perfil profesional }\end{array}$ & $\begin{array}{l}\text { Pertinente y acorde a } \\
\text { su experiencia }\end{array}$ & $\begin{array}{l}\text { Pertinente y } \\
\text { acorde a su } \\
\text { experiencia }\end{array}$ \\
\hline $\begin{array}{l}\text { activa: } \\
\text { isión del } \\
\text { de } \\
\text { idad }\end{array}$ & $\begin{array}{l}\text { Básico con apoyo de } \\
\text { modelos didácticos }\end{array}$ & $\begin{array}{l}\text { eModerado } \\
\text { con poco manejo } \\
\text { didáctico }\end{array}$ & $\begin{array}{l}\text { Básico con apoyo de } \\
\text { estrategias } \\
\text { experienciales }\end{array}$ & $\begin{array}{l}\text { Básico con apoyo } \\
\text { de la experiencia }\end{array}$ \\
\hline
\end{tabular}

Fuente: Elaborado por los autores (2020)

De manera grupal, este estudio permitió inferir que, el PCK de los maestros se afectó por las fuentes de su conocimiento probabilístico y didáctico, quienes recibieron más cursos que incluyeron el tema de probabilidad resultaron más robustos en la componente del PCK referido al conocimiento del contenido, mientras que aquellos que han acumulado un mayor número de estrategias atribuibles a su experiencia, salieron fortalecidos en la componente del PCK asociada con las estrategias instruccionales. Por otra parte, su básico desarrollo del PCK puede atribuirse a sus concepciones sobre la probabilidad, las cuales prevalecieron en el plano intuitivo o en el clásico pero con poca posibilidad de trascender hacia la probabilidad axiomática en espacios muestrales infinitos, el hecho de que el docente haya tratado de aplicar el concepto de equiprobabilidad en este tipo de espacios también refleja bajos niveles de comprensión del concepto de probabilidad, situaciones semejantes fueron detectadas en el estudio reportado por Thibault \& martin (2018). Finalmente; su conocimiento del currículo tiene una relación directa con su perfil profesional y los años de experiencia docente, aspectos que además afectan el desarrollo del PCK de los docentes.

De la fase post-activa emergieron dos categorías: postura en torno a dos situaciones problema sobre probabilidad y la reflexión docente en torno a las fases pre-activa e interactiva. En este sentido, la primera situación problema que se les planteó fue: se lanzan simultáneamente dos dados equilibrados ¿cuál de las siguientes opciones tiene mayor probabilidad de ocurrir? i) obtener un cinco y un seis, ii) obtener el par $(6,6)$, iii) ambas tienen la misma probabilidad, iv) no sé (Lecoutre \& Cordier, 1990). La segunda situación explora el cálculo de probabilidades en un espacio muestral infinito, se planteó así: en el espacio muestral conformado por los números naturales $(\mathrm{N})$, se toma el sigma álgebra conformado por el conjunto parte de $\mathrm{N}$ y se define la siguiente función para medir probabilidades en $\mathrm{N}$ (Burbano \& Valdivieso, 2015): $\mathrm{P}(n)=2^{-n}$ (dos a la menos $n$ ) con $n=1,2,3, \ldots$ para los eventos $E=\{1,3,5, \ldots\}$ y $F=\{2,4,6, \ldots\}$ correspondientes a los números impares y pares respectivamente, la probabilidad de ocurrencia del evento $E$ es, i) un tercio, ii) un medio, igual que la probabilidad de $F$, iii) dos tercios, iv) no lo sé. En la primera 
situación tres docentes respondieron acertadamente con la opción i); sin embargo, uno de ellos consideró que la opción iii) era la correcta; en consecuencia, la mayoría de los profesores reflejó un conocimiento probabilístico pertinente basado en la concepción clásica. En la segunda, solamente un profesor respondió con acierto al elegir la opción iii) y los demás, seleccionaron la opción ii) la cual es incorrecta.

Estos hallazgos permitieron interpretar que, el conocimiento del contenido probabilístico de los participantes aún es parcializado, su estructura cognitiva refleja bajos niveles de comprensión del concepto de probabilidad, su PCK en probabilidad refleja fortalezas en cuanto al uso de la concepción clásica en espacios finitos, en un alto porcentaje y con grandes posibilidades para corregir el sesgo de equiprobabilidad en sus estudiantes; por otra parte, los docentes reflejan una baja elaboración del concepto de probabilidad en espacios muestrales infinitos desde la concepción axiomática e intentan aplicar su concepto de equiprobabilidad en espacios infinitos de manera poco reflexiva.

Finalmente, en la reflexión docente en torno a las fases pre-activa e interactiva, los profesores manifiestan que, en cierta medida, ellos enseñan el tema de probabilidad de forma semejante a como se lo transmitieron en la universidad pero de acuerdo con las directrices ofrecidas por el MEN en el currículo de matemáticas, desarrollan sus clases fundamentados en alguno de los enfoques pedagógicos actuales como el constructivismo, el aprendizaje significativo y la resolución de problemas, entre otros, pero sobre todo lo hacen convencidos de que la probabilidad y las matemáticas en general se han de enseñar de manera más humana y menos impositiva; también consideran que, el tema es difícil de comprender a cabalidad y de enseñar, requiriéndose mayores niveles de formación al recibido en la universidad tanto desde la parte disciplinar como de la didáctica, el docente así planifique y ejecute bien sus clases no está exento de errores y sesgos, adicionalmente, el maestro hace lo mejor que puede con el conocimiento que tiene y necesita capacitarse continuamente en temas de alta complejidad como el de la probabilidad (Vásquez y Alsina, 2019).

\section{Discusiones y conclusiones}

La exploración realizada en este trabajo ha evidenciado que se requiere acrecentar el PCK de los docentes de probabilidad que ofrecen el tema en la educación media a fin de que preparen mejor a sus estudiantes respecto al contenido probabilístico necesario para proseguir con éxito en los cursos universitarios que lo incluyen en diversas titulaciones; en este sentido, Ibáñez y Villasana (2020) sugieren diseñar propuestas didácticas que posibiliten suplir las falencias presentes en los procesos instruccionales realizados por los profesores de estas y otras materias a nivel universitario. Además, Ernst \& Morton (2020) recomiendan la elaboración de un abordaje progresivo del tema de probabilidad en el currículo escolar. 
Los resultados evidencia que, aunque con algunas imprecisiones en el uso amplio y complejo del concepto de probabilidad y un marcado énfasis en la concepción clásica (laplaciana) más que en las otras, el componente del PCK más desarrollado de los docentes corresponde al conocimiento del tema por enseñar; este hecho guarda relación con lo señalado por Sánchez y Monroy (2013) en el sentido de que esta concepción es la que se ha privilegiado en la enseñanza de la probabilidad a nivel pre universitario y universitario, concepción que según Rodríguez (2021) también prevalece en los libros de texto. Adicionalmente, estudios como el de Pierce \& Chick (2011) enfatizan en que a nivel mundial, un gran número de maestros poseen poco conocimiento acerca de la probabilidad y de didácticas específicas para enseñarla. Las componentes del PCK con menor desarrollo son las referidas al uso de estrategias instruccionales y a la forma cómo el estudiante aprende; al respecto, los docentes usan un número reducido de estrategias de tipo general como los ejemplos, ejercicios, problemas tipo, y algunas específicas como los juegos de azar, dados, monedas y el diagrama de árbol (Chernoff \& Zazkis, 2011), entre otros; centradas en el cálculo de la probabilidad para eventos finitos; sin lograr trascender hacia los espacios infinitos con medidas de probabilidad que podrían ejemplificarse con el uso de progresiones geométricas como se explicita en Burbano y Valdivieso (2020) para algunos casos o en el uso de razonamiento inductivo y deductivo como se menciona en Salazar, Botero y Giraldo (2020).

En cuanto a la capacidad de los maestros del grupo de estudio para corregir algunas heurísticas y sesgos en sus estudiantes se puede decir que es moderada, en tanto que en ellos también están latentes ciertas heurísticas y sesgos probabilísticos (Yetim, 2019); en este sentido, durante la práctica pedagógica se observaron dificultades para responder asertivamente casos puntuales de preguntas formuladas por los estudiantes y una potencial presencia del sesgo de equiprobabilidad en el intento de extenderla hacia espacios infinitos con poca reflexión; en este contexto, cobra vigencia la afirmación de Carlsen (1987) de que cuando el maestro no conoce a profundidad el tema de una lección, puede limitar las intervenciones de sus estudiantes y explicitar poca capacidad para responderles adecuadamente. Además, esto ratifica lo expresado por los participantes en la fase posactiva en referencia a que la probabilidad es un tema difícil de enseñar, aspecto también expresado en el trabajo realizado por Godino, Batanero, Roa \& Wilhelmi (2008) y Sriraman \& Chernoff (2020), aún más cuando en el aula se trata de integrar los significados de probabilidad clásica, frecuencial y axiomática expuestos por Batanero (2020). A pesar de estas dificultades, se rescata la disposición de los profesores para motivar a sus estudiantes y hacer el tema interesante, agradable y menos dificultoso para los alumnos al interactuar con los juegos de azar.

La triangulación de información posibilitó corroborar la presencia de poco desarrollo del PCK en el grupo de estudio y una baja comprensión del concepto de probabilidad; los resultados sobre tal comprensión guardan relación con los obtenidos por Inzunsa y Guzmán (2011) en referencia a profesores de secundaria mexicanos, quienes manifestaron que la 
probabilidad era un área complicada para trabajar y requería altos niveles de comprensión. Por otro lado, la práctica pedagógica de los docentes se caracterizó por la presencia de un enfoque personal fundamentado en las concepciones implícitas en el profesor (Tzur, Simon, Heinz \& Kinzel, 2001), en teorías sobre el aprendizaje estudiantil y su experiencia docente para interactuar de la mejor manera posible con sus alumnos, esta práctica presenta rasgos didácticos diversos pero que contrasta con las secuencias tradicionales para enseñar matemáticas basadas en modelos trasmisionistas (Zapata, 2011; Burbano, 2017), dado que la acción pedagógica con el tema de probabilidad ha de incluir el carácter contra intuitivo del mismo y un alto desarrollo del PKC del profesor (Burbano, et al., 2017; Batanero, 2020). Finalmente, resulta razonable que tanto desde el MEN como de las instituciones educativas se promueva el desarrollo del PCK en sus docentes por tratarse de una manera holística de pensar, comprender y razonar del profesor, destinada a mejorar la forma de enseñar determinados temas del currículo escolar; actualmente el PCK se puede incrementar con el uso de las tecnologías de la información y las comunicaciones (TIC) y adentrarse en el modelo TPACK (Chai, et al., 2019) para de ese modo acceder a estrategias instruccionales alternativas como la simulación de eventos y variables aleatorias (Rodríguez \& Ramírez, 2018) o el trabajo soportado en ambientes virtuales y didácticas específicas (Mendoza, Burbano \& Valdivieso, 2019).

Este trabajo investigativo permitió establecer las siguientes conclusiones:

Con base en el análisis de contenido realizado sobre los datos textuales se evidenció la emergencia de tres categorías de conocimiento requeridas por el docente para enseñar probabilidad: i) dominio del contenido o tema por enseñar, en esta categoría los docentes presentan un conocimiento básico del tema de probabilidad anclado en la concepción clásica de Laplace prioritariamente, ii) uso de estrategias instruccionales pertinentes aunque no suficientes, al respecto los docentes utilizan un variado repertorio de representaciones instruccionales como los juegos de azar con dados y monedas, tablas de frecuencias, diagramas de Venn y de árbol, ejemplificaciones, preguntas orientadores, y trabajo grupal, entre otras, focalizadas en espacios muestrales finitos y la equiprobabilidad sin trascender a espacios infinitos, y iii) conocimiento sobre cómo el estudiante aprende el tema de probabilidad, en este sentido, los profesores presentaron una capacidad moderada para detectar y corregir ciertas concepciones erróneas y algunos sesgos probabilísticos en sus estudiantes y en ellos mismos. La manera cómo los docentes conjugaron estas tres categorías en su práctica pedagógica reflejaron poco desarrollo de su PCK y baja comprensión del concepto de probabilidad.

Tanto las concepciones de los profesores como otros aspectos asociados con su conocimiento del contenido probabilístico, las didácticas específicas y los modelos pedagógicos implícitos en el docente están relacionados con el nivel de desarrollo de su PCK; por ejemplo, en su concepción sobre el aprendizaje de la probabilidad, los casos 1 y 2 incorporan elementos del constructivismo y resolución de problemas, mientras los casos 3 y 4 lo hacen con el aprendizaje significativo y la resolución de problemas respectivamente. 
Estos aspectos inciden sobre su forma de implementar las actividades y de llevar a cabo los procesos de interacción con sus estudiantes en la clase, la forma de plantear los trabajos en grupo, la discusión en el aula y la atención a los estudiantes realizada en distintos momentos.

En la interacción de cada profesor con sus estudiantes se hacen visibles y 'se confirman algunas relaciones existentes entre las concepciones del profesor sobre la probabilidad su enseñanza y aprendizaje, y su PCK'; por ejemplo, el concepto de probabilidad se enseña de forma intuitiva usando representaciones instruccionales basadas en los juegos de azar y cálculo de la probabilidad clásica sin transitar hacia los elementos del concepto de manera formal o axiomática requerida para avanzar más allá de la equiprobabilidad e incursionar en la medición de probabilidades sobre espacios muestrales infinitos.

La formación del docente durante su pregrado, la auto-capacitación y el contexto escolar donde ejerce su práctica docente influyen sobre el tema por enseñar o el conocimiento del contenido probabilístico como una de las componentes de su PCK; además, las estrategias instruccionales utilizadas por los profesores en la clase y su experiencia docente se constituyen en factores que hacen a los maestros únicos en ciertos aspectos referentes a su PCK en la práctica pedagógica en los cuatro casos involucrados en el estudio, focalizados en la forma como ellos integran el concepto de probabilidad centrado en las concepciones intuitiva, clásica, frecuencial y axiomática.

\section{Referencias}

Appova, A., \& Taylor, C. E. (2020). Providing opportunities to develop prospective teachers' pedagogical content knowledge. In A. Appova, R. M. Welder, and Z. Feldman, (Eds.), Supporting Mathematics Teacher Educators' Knowledge and Practices for Teaching Content to Prospective (Grades K-8) Teachers. The Mathematics Enthusiast. vol. 17, nos. 2 \& 3, pp. 673-724. ScholarWorks: University of Montana. https://scholarworks.umt.edu/tme

Ball, D. L., Thames, M., \& Phelps, G. (2008). Content knowledge for teaching: What makesit special? Journal of Teacher Education, 59(5), 389-407.

Bardín, L. (1991). El análisis de contenido. Madrid, España: Akal.

Batanero, C. (2020). Probability Teaching and Learning. In: Lerman S. (eds) Encyclopedia Of Mathematics Education. Springer. https://doi.org/10.1007/978-3-030-15789-0_128

Babbie, E. R. (2020). The practice of social research. Cengage learning. 
Burbano, V., \& Valdivieso, M. (2015). Elementos de probabilidad. Apoyo al estudio independiente. Editorial UPTC.

Burbano, V. (2017). Un acercamiento a la didáctica de la probabilidad desde el PCK para el nivel pre universitario. Editorial UPTC.

Burbano, V. M. Á., Valdivieso, M. A., \& Aldana, E. (2017). Conocimiento base para la enseñanza: un marco aplicable en la didáctica de la probabilidad. Revista de Investigación, Desarrollo e Innovación, 269-285. https://doi.org/10.19053/20278306.v7.n2.2017.6070

Burbano, V., \& Valdivieso, M. (2020). Una mirada histórica de las medidas de probabilidad reales desde la investigación documental. Editorial UPTC.

Callingham, R., \& Watson, J. (2011). Measuring levels of statistical pedagogical content knowledge. En C. Batanero, G. Burrill, y C. Reading (Eds.), Teaching statistics in school mathematics. Challenges for teaching and teacher education. A joint ICMI and IASE study (pp. 283-293). Springer.

Carlsen, W. (1987). Why Do you Ask? The Effects of Science Teacher Subject Matter Knowledge on Teacher Questioning and Classroom Discourse, paper presented at the annual meeting of the American Educational Research Association. ERIC Number: ED293181

Chai, C.S., Jong, M. S., Yin, H., Chen, M., \& Zhou, W. (2019). Validating and Modelling Teachers' Technological Pedagogical Content Knowledge for Integrative Science, Technology, Engineering and Mathematics Education. Educational Technology \& Society, 22(3), 61-73. https://doi.org/10.2307/26896710

Chernoff, E. J., \& Zazkis, R. (2011). From personal to conventional probabilities: from sample set to sample space. Educational Studies in Mathematics, 77(1), 15-33. https://doi.org/10.1007/s10649-010-9288-8

Colestock, A., \& Sherin, M. (2009). Teachers' sense-making strategies while watching Video of mathematics instruction. Journal of Technology and Teacher Education, 17(1), 7-29. https://www.learntechlib.org/primary/p/26234/

Even, R., \& Kvatinsky, T. (2010). What mathematics do teachers with contrasting teaching approaches address in probability lessons? Educational Studies in Mathematics, (74), 207222. https://doi.org/10.1007/s10649-010-9234-9

Ernst, H., \& Morton, A. (2020). Probability across the curriculum. Australian Mathematics Education Journal, 2(2), 4-7. https://eric.ed.gov/?id=EJ1261849 
Gadamer, H. G. (1976). El círculo hermenéutico y el problema de los prejuicios. Suhrkamp:

Godino, J. D., Batanero, C., Roa, R., \& Wilhelmi, M. R. (2008). Assessing and developing

pedagogical content and statistical knowledge of primary school teachers through project work.

En C. Batanero, G. Burrill, C. Reading \& A. Rossman (Eds.), Joint ICMI/IASE Study: Teaching Statistics in School Mathematics. Challenges for Teaching and Teacher Education. Proceedings of the ICMI Study 18 and 2008 IASE Round Table Conference. ICMI and IASE.

Godino, J. D. (2009). Categorías de análisis de los conocimientos del profesor de matemáticas [Categories for analysing the knowledge of mathematics teachers]. Revista Iberoamericana de Educación Matemática, (20), 13-31. http://hdl. handle.net/123456789/3098

Gómez, E. (2014). Evaluación y desarrollo del conocimiento matemático para enseñar la probabilidad en futuros profesores de educación primaria. [Tesis Doctoral, Universidad de Granada, España].

Hacking, I. (1995). El Surgimiento de la Probabilidad. Gedisa.

Hernández, R., \& Mendoza, C (2018). Metodología de la investigación. Las rutas cuantitativa, cualitativa y mixta. Editorial Mc Graw Hill.

Ibáñez, R., \& Villasana, P. (2020). Aproximación crítica al conocimiento didáctico del contenido en educación superior y sus posibilidades de estudio. Revista Espacios, 41(18), 21-28.

Inzunsa, S., \& Guzmán, M. (2011). Comprensión que muestran profesores de secundaria acerca de los conceptos de probabilidad: un estudio exploratorio. Educación Matemática, $23(1), 63-95$.

Jackson, P. (1975). La vida en las aulas. Madrid. Ediciones Marova.

Jones, G., Langrall, C., Thornton, C., \& Mogill, T. (1997). A framework for assessing and nurturing young children's thinking in probability. Educational Studies in Mathematics, (32), $101-125$.

Kolmogórov, A. (1956). Foundations of the theory of probability. Chelsea.

Laplace, P. S. (1812). Théorie analytique des probabilités. Mme Ve Courcier. 
Lave, J., \& Wenger, E. (1991). Situated learning. Legitimate Peripherals participation. University Press.

Le Compte, M. D., \& Goetz, J. P. (1982). Problems of reliability and validity in ethnographic research. Review of educational research, 52(1), 31-60.

Lecoutre, M. P., \& Cordier, J. (1990). Effect du mode de présentation d'un problème Aleatoire sur les modèles développés par les élèves. Bulletin de I'APMEP, 372, pp. 9-22.

Llinares, S. (2000). Intentando comprender la práctica del profesor de matemáticas. En J.

Ponte \& Serrazina, L. (Eds.) Educaçao Matemática em Portugal, Espanha e Italia. Actas da Escola de Verao-1999 (pp. 109-132). Sociedade de Educaçao Matemática da Sociedade Portuguesa de Ciencias de Educaçao.

Martin, V., Héroux, S., Homier, M., \& Thibault, M. (2021). L'analyse de tâches probabilistes proposées dans des cahiers d'apprentissage destinés à l'enseignement-apprentissage des mathématiques au primaire au Québec: exemplification de tâches inscrites dans l'approche fréquentielle. Canadian Journal of Science, Mathematics and Technology Education, 21(1), 145-165. https://doi.org/10.1007/s42330-021-00134-w

Mendoza, H., Burbano, V., \& Valdivieso, M. (2019). El papel del docente de matemáticas en

Educación superior a distancia y virtual: una mirada desde los métodos mixtos de investigación. Revista Espacios, 40(39), 3-15.

Ministerio de Educación Nacional de Colombia -MEN-. (2003). Estándares básicos de competencias en matemáticas. Santafé de Bogotá. www.mineducacion.gov.co/

Mises, R. (1952). Probabilidad, estadística y verdad. Espasa-Calpe.

National Council of Teachers of Mathematics -NCTM-. (2000). Principles and standard for school mathematics. NCTM.

Gavira, S. A., \& Osuna, J. B. (2015). La triangulación de datos como estrategia en investigación educativa. Pixel-bit. Revista de medios y educación, (47), 73-88.

Pierce, R., \& Chick, H. (2011). Teachers'beliefs about statistics education. En C. Batanero, G. Burrill y C. Reading (Eds.), Teaching statistics in school mathematics- Challenges for teaching and teacher education (pp. 151-162). Springer.

Pincheira, N., Vásquez, C., \& Giacomone, B. (2021). Una aproximación al conocimiento didáctico-matemático de futuros profesores de Educación Básica para enseñar matemáticas 
elementales. Uniciencia, 35(2), 1-22.

Pinto, J. (2010). Conocimiento didáctico del contenido sobre la representación de datos estadísticos: estudio de casos con profesores de estadística de las carreras de psicología y educación. [Tesis doctoral, Universidad de Salamanca, España].

Ponte, J. P., Quaresma, M., \& Branco, N. (2012). Práticas profissionais dos professores de

Matemática. Avances de Investigación en Educación Matemática, (1), 65-86.

Rodríguez, G., Gil, J., y García, E. (1999). Metodología de la investigación cualitativa. Ediciones Aljibe.

Rodríguez, J. E. (2021). Análisis Ontosemiótico de dos libros de texto colombianos de grado quinto: el caso del objeto matemático probabilidad. [Trabajo de grado de Maestría, Universidad Pedagógica y Tecnológica de Colombia].

Rodríguez, K., \& Ramírez, G. (2018). Simulación de variables aleatorias continuas y el teorema del límite central. Revista digital Matemática, Educación e Internet, 18(1), 1-24.

Salazar, C., Botero, D., \&_Giraldo, L. (2020). Enseñanza y Aprendizaje del Razonamiento Deductivo e Inductivo mediante las Ciencias Naturales. Educación y Humanismo, 22(38), 118. https://doi.org/10.17081/eduhum.22.38.3732

Sánchez, M. (2011). A review of research trends in mathematics teacher education. PNA Revista de Investigación en Didáctica de la Matemática, 5(4), 129-145.

Sánchez, E. S., \& Monroy, J. C. V. (2013). La cuantificación del azar: una articulación de las definiciones subjetiva, frecuencial y clásica de probabilidad. Revista de didáctica de la Estadística, (2), 39-46.

Schoenfeld, A. H., \& Kilpatrick, J. (2008). Toward a theory of proficiency in teaching mathematics. En D. Tirosh y T. Wood (Eds.), Tools and processes in mathematics teacher education (pp. 321-354). Sense Publishers.

Shulman, L. S. (1986). Those who understand: Knowledge growth in teaching. Educational Research, 15(2), 4-14.

Shulman, L. S. (1987). Knowledge and teaching: foundations of the new reforms. Harvard Educational Review, 57(1), 1-22.

Simons, H. (2011). El estudio de caso: Teoría y práctica. Morata. 
Sriraman, B., \& Chernoff, E. J. (2020). Probabilistic and Statistical Thinking. In: Lerman S.

(eds) Encyclopedia of Mathematics Education. Springer. https://doi.org/10.1007/978-3-03015789-0_100003

Stake, R. (1998). Investigación con estudio de casos. (2a. ed.). Morata.

Strauss, A., \& Corbin, J. (1998). Basics of qualitative research. Techniques and procedures for developing grounded theory. Sage publications, Inc.

Swenson, K. A. (1998). Middle school mathematics teachers' subject matter knowledge and pedagogical content knowledge of probability: its relations to probability instrution. [Disertación Doctoral, Universidad del Estado de Oregon].

Taylor, S., \& Bogdan, R. (1987). Introducción a los métodos cualitativos de investigación. Paidós.

Thibault, M., \& Martin, V. (2018). Confusion autour du concept de probabilité. For the learning of mathematics, 38(1), 12-16.

Torres, J., Cifuentes, J., y Espejo, R. (2020). Pedagogical Trends in Teaching

Humanities in the Formation of Future Elementary School Teachers. Educación y Humanismo, 22(38), 1-16. https://doi.org/10.17081/eduhum.22.38.3628

Tzur, R., Simon, M. A., Heinz, K., \& Kinzel, M. (2001). An account of a teacher's Perspective on learning and teaching mathematics: Implications for teacher development. Journal of Mathematics Teacher Education, 4(3), 227-254.

Unangst, J., Amaya, A. E., Sanders, H. L., Howard, J., Ferrell, A., Karon, S., \& Dever, J. A. (2020). A process for decomposing total survey error in probability and nonprobability surveys: A case study comparing health statistics in US internet panels. Journal of Survey Statistics and Methodology, 8(1), 62-88.

Vásquez, C. A. (2014). Evaluación de los conocimientos didáctico-matemáticos para la enseñanza de la probabilidad de los profesores de educación primaria en activo. [Tesis doctoral, Universidad de Girona, España].

Vásquez, O. C., \& Alsina, Á. (2019). Conocimiento especializado del profesorado de educación básica para la enseñanza de la probabilidad. Profesorado, Revista de Currículum y $\begin{array}{llll}\text { Formación del Profesorado, 393-419. } & \text { 23(1), }\end{array}$ https://doi.org/10.30827/profesorado.v23i1.9160

Venkat, H., \& Adler, J. (2020). Pedagogical Content Knowledge Within "Mathematical 
Knowledge for Teaching". In: Lerman S. (eds) Encyclopedia of Mathematics Education. Springer, Cham. https://doi.org/10.1007/978-3-030-15789-0_123

Watson, J. M. (2001). Profiling teachers' competence and confidence to teach particular mathematics topics: The case of data and chance. Journal of Mathematics Teacher Education, (4), 305-337.

Yetim, S. (2019). Mistakes and Misconceptions of Middle School Students about

Probability: A Concept Map Study. Bartın University Journal of Faculty of Education, 8(1), 5481. https://doi.org/10.14686/buefad.427971

Yin, R. K. (2014). Case Study Research: Design and Methods (5 Ed.). Sage publicatios.

Zapata, L. (2011). ¿Cómo contribuir a la alfabetización estadística? Revista Virtual Universidad Católica del Norte, 33(1), 234-247.

Financiación: Este artículo de investigación se derivó del proyecto "Conocimientos del profesor para la enseñanza de la probabilidad: estudio de casos con profesores en ejercicio" con código SGI 1748. 